\title{
The Summary of Internet of Things Applications in the Field of Pump Monitoring
}

\author{
Fenglian $\mathrm{Qi}^{\mathrm{a}}$, Guancheng Liu ${ }^{\mathrm{b}}$ \\ Shenyang Jianzhu University \\ a614582608@qq.com, ${ }^{\text {b1416959573@qq.com }}$
}

\begin{abstract}
Keywords: Internet of things (IoT), Pump, Monitoring, Summarize
Abstract. With the progress of science and technology, Internet of things (IoT) technology is developing rapidly. At the same time, pump equipment is widely used in many fields. The development status and trends of the Internet of things (IoT), current situation of pump equipment monitoring, and application of Internet of things (IoT) in pump equipment monitoring are introduced in detail based on a large number of document literature in this paper. This paper focuses on the analysis and description the deficiencies of current pump equipment monitoring, and put forward the trend plan for improving the service life and the operation monitoring supervision mechanism of the pump by combining the advantages of intelligent Internet of things technology. This paper has a certain guiding role for the development of pump equipment monitoring.
\end{abstract}

\section{Development Status and Development Trend of Internet of Things (IoT)}

\section{Definition of Internet of Things (IoT)}

In 1999, the Massachusetts Institute of Technology proposed the concept of Internet of things (IoT). At present, there is a common public interpretation for Internet of things (IoT). Internet of things (IOT) is a kind of network technology, which connects the information sensing equipment (such as RFID, laser scanner, GPS, and other equipment) with a variety of wireless communication equipment, and realizes intelligent monitoring and positioning through the exchange of information. Generally speaking, Internet of things (IoT) is a kind of comprehensive technology that connects things with other things through network to make things easy to control and manage, and serve the development of society better ${ }^{[1]}$. We can also define Internet of things (IoT) for the evolution of the Internet. It's the Internet that contains billions of sensors, cameras, wearable, smart phones and other intelligent networking equipment.

Compared with Internet, Internet of things (IOT) has its unique nature:

(1) Extensive and comprehensive sensor technology.

(2) Identifiable RFID Tags.

(3) Convenient and flexible embedded system technology.

\section{Development Status of Internet of Things}

The rapid development of Internet technology makes the world into the information era. The wide application of information technology not only changes people's way of life, but also promotes the progress and development of society in various fields. With its many advantages, Internet of things (IoT) achieve the perfect fusion with Internet, which not only extends the functionality of network system, but also has a role in promoting development of society and economy ${ }^{[2][3]}$. In 2016, global networking equipment valued 6.4 billion dollars have been used (30\% higher than 2015). That is to say, new IoT equipment which value 5.5 million dollars was produced and used every day in 2016 . In addition, the IoT market has a \$235 billion's expenditure for the services of the government in 2016 (22\% higher than 2015). From the perspective of hardware spending, consumption in 2016 reached $\$ 546$ billion. Also, the expenditure of intelligent service and other applications used to connect equipment reached $\$ 868$ billion in enterprises. 
The development of the Internet of things is mainly divided into three stages:

The first phase is the phase of a large number of equipment connections. IoT connections were established on a large scale at this phase. The core of this phase is infrastructure construction, connection construction and management, terminal's intelligence of network.

The second phase is the phase of perception and initial formation of big data. A large number of equipment connected to the network are perceived at this phase, resulting in massive big data. At the same time, cloud computing developed rapidly accompanied by IoT.

The third phase is the phase of initial intelligent formation. The initial artificial intelligence gradually realized at this phase. Intelligent analysis, applications and services of IoT data became commercialized through self-learning and intelligently two-way linkage, and maximized the value of the data ${ }^{[4][5]}$.

With the development of IoT, the IoT technology has been applied to many fields of production and life:

Intelligent Transportation. IoT makes cities become smart cities. It connects Internet with roadside sensors and on-board equipment to carry out related operations, which is helpful to relieve traffic pressure. Such as, roadside sensors which are deployed on busy highways can measure speed by tracking car drivers' mobile phone traffic. Inserting the OBDLink MX WiFi devices into the OBD-II port of the car to turn the smartphone into a complex scanning tool to monitor car's performance at real time, and track your driving habits ${ }^{[6]}$. Volvo's autopilot is also a good manifestation of the application of $\mathrm{IoT}^{[7]}$.

Digital Home. You can direct home appliances in your office with IoT technology. On your way home from work, meals have been cooked, hot water for bathing has been ready and personalized TV programs will be broadcast on time. Family facilities can be automatically noticed to repair at ordinary times. A large number of such family equipment connect with external services through IoT, and truly realize the interaction between services and equipment ${ }^{[8]}$.

Modern Logistics Management. Through IoT technology, every link in the supply chain can be accurately perceived and mastered by implanting sensor chip (node) in products, including purchasing, manufacturing, packaging/loading and uploading, stack, transportation, distribution, sale and service. Then, combining the perceived information with the GIS/GPS database in the background, the logistics information is integrated into a powerful information network, which is helpful for the management of logistics and the tracking of goods.

Intelligent Production. In the industry, combined with the Internet of things, making all kinds of terminal with the ability to perceive the environment, computing model based on the ubiquitous technology and mobile communication into every link of industrial production can greatly increase productivity, improve product quality, and reduce product cost and resource consumption. IoT promotes the traditional industry to a new phase of intelligent industry.

Digital Medical. Automatic identification technology (RFID as the representative of it) can help hospitals achieve the uninterrupted monitoring and diagnosis for patients, the sharing of medical records, and the tracking of medical equipment to improve the patient's health ${ }^{[9]}$. For example, smart pills can let the doctor monitor the medication time and dose ${ }^{[10]}$. IoT extends this service to the whole world through the Internet. The integration of IoT with hospital information system and drug logistics system is the inevitable trend for medical informatization.

Food Safety Control. Through the label recognition and IoT technology, we can monitor production process of food, track the quality of food, and effectively prevent safety accidents of food anywhere and anytime. IoT greatly improves the safety management level of food.

IoT also has good application in power, agriculture and other fields. For example, Smart meter is the device that can build Internet connection. It allows people reading and analysis power consumption and power demand, and then analyzes the needs of customers and power distributors based on the data collected. In agricultural, such as the virtual fence device, it can improve the utilization rate of the ranch and keeps sick animals away from the animal health animals to prevent the spread of the disease. 


\section{Present Situation of Pump Equipment Monitoring}

\section{Overview of Pump Equipment}

A pump is a machine that converts the mechanical energy of the prime mover into liquid energy, which is used to increase potential energy, pressure energy, or kinetic energy (high speed liquid energy) of the liquid. Usually, the prime mover (motor, diesel engine) rotates the impeller through the pump shaft, and works on the liquid to increase its energy. Thus, the required amount of liquid is transported to the required height or the place of required pressure through the pump's flow parts from the suction tank.

Nowadays, pump equipment has been applied to various fields according to their functional requirements:

Used in Agriculture. As the machinery of irrigation and drainage, pumps are used to ensure the normal growth of crops to ensure agricultural production

Applied to the Chemical Industry Field and the Petroleum Production Field. Pumps transport liquid and provide pressure flow of chemical reaction for complex process of this field.

Used in Mining and Metallurgical Industries. Pumps are used for water supply and drainage in the process of ore dressing, smelting and rolling.

Applied in the Electric Field. Especially in nuclear power stations, nuclear main pump, two pumps, three pumps are required to apply in there. And, feed-water pumps, condensate pumps, circulating pumps and ash pumps are widely used in thermal power plant.

Widely Used in Shipbuilding Industry. Such as pumps used in ocean going wheels.

Used in National Defense Construction. Pumps are used to regulate the flap, tail rudder and landing gear of the aircraft in this filed. The rotation of warships and tank turrets, and the ups and downs of submarine also need to use pumps.

Pumps are also used in urban water supply and drainage, transportation of bleaching liquid and dyes in textile industry, pulp transportation in paper industry, and food industry.

\section{Monitoring Status of Pump Equipment}

Before 1990s, the real-time monitoring system of pump equipment is traditional, closed, so it's very difficult to upgrade the system. In recent years, with the rapid development of computer RISC technology, interface technology, network technology, and standardized operating system technology, monitoring level of pump equipment is greatly improved ${ }^{[11]}$.

After years of development, pump monitoring can be divided into three monitoring modes:

Traditional Monitoring Mode. The traditional mode is the artificial mode, only adding some instruments on the pump equipment to identify the current state of the pump. People regularly check the equipment parameters. In many cases, pumps can only be checked in the event of equipment failure. Although this mode is less capital investment, the damage to pump equipment is larger, and it has a greater impact on the operation of pump equipment.

Controller Single Monitoring Mode. This mode uses PLC as the control core, and uses the touch screen and the control panel as an interface to realize the real-time monitoring of operation data of pump equipment, complete the basic data acquisition and control functions. Compared with the traditional model, this mode has made great progress and has better performance in improving the operation supervision and service life of pump equipment.

Based on the Central Controller Monitoring Mode. This mode makes use of PLC, PAC or PC as the control core. By using the advantages of computer data processing, it can not only complete basic data acquisition and control functions, but also complete fault diagnosis, data analysis, report formation and printing. At the same time, it also can exchange data with management to provide operators with flexible and convenient man-machine interface. This mode is currently used pump equipment monitoring mode at home and abroad. Figure. 1 is the overall structure of monitoring 
system for this mode.

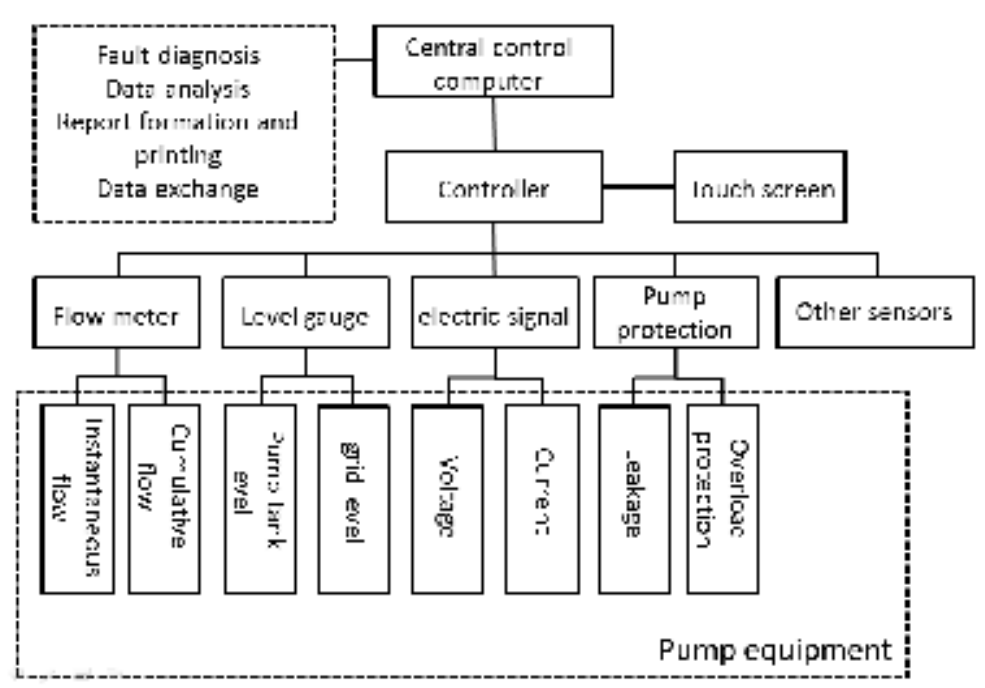

Figure.1 Overall Structure of Monitoring System

Taking typical water pumping station as an example, computer monitoring has been used abroad to monitor the operating conditions of pumping stations and do centralized management of all pumping stations in the whole basin. Its functions include pump operation monitoring, operation fault diagnosis, multi-layer control and so on. Multimedia technology has been applied to the monitoring system $^{[12]}$.

In recent years, most of the domestic large pump stations have set up a microcomputer data acquisition system, but compared to the monitoring technology of pump equipment abroad, the domestic pump monitoring technology is not mature enough. Under normal circumstances, the parameters of the pump can only be monitored and alarmed, and the running pumps can not be controlled by computer. Therefore, it is necessary to enhance the improvement of pump monitoring technology in operation parameters and operating conditions, and keep up with the international situation of intelligence. There are deficiencies mainly in the following aspects:

Real Time is Not Enough. With the progress of technology, the monitoring of pump equipment has become more and more demanding for the parameters. Due to the limitation of computer processing speed, a large amount of data will lead to the slowdown of the data processing and the real time of the pump equipment monitoring can be influenced. At the same time, the performance analysis of each parameter of the pump is restricted ${ }^{[13]}$.

Small Amount of Data. The storage capacity of the local database leads to the failure to monitor all parameters of pumps in a very comprehensive and real-time manner, affecting the maintenance of pump parameters throughout its life cycle.

Software Lacks Sharing. Nowadays, the functions of monitoring software are very similar, but there are differences between the monitored objects, also the hardware. This makes the software's monitoring interface and control algorithms need to be reprogrammed. Therefore, the software's sharing needs to be improved ${ }^{[14]}$.

Poor Data Sharing. Currently, pump equipment is rapidly being replaced and data sharing between manufacturers, vendors and users is required to maintain and optimize pump performance. However, at this stage of the monitoring system can not achieve the sharing of data, thus affecting the pump equipment optimization.

Intelligent Level is Low. Intelligent monitoring system can send the operating parameters and real-time status of pumps to the fault management system, given treatment measures to troubleshoot. This is an area that many domestic monitoring systems urgently need to improve. 


\section{IoT-based pump equipment monitoring trend}

\section{development trend of Internet of Things (IoT)}

American Gartner company forecasts ${ }^{[15]}$, the Global IoT equipment usage will reach $\$ 20.8$ billion in 2020. Gartner also pointed out that spending of global IoT hardware and intelligent services used in connected services in the enterprise use will reach 1.5440 billion US dollars and 1.4770 trillion US dollars in 2020. The sources of other aspects are even more numerous. We can narrate the development trend of IoT in the following aspects:

The Combination of IoT with Hardware. With the development of IoT, a variety of sensors and monitoring equipment are included in the hardware equipment to make the hardware more intelligent. At the same time, mobile equipment can monitor hardware remotely and analyse instruction intelligently relying on the network ${ }^{[16]}$. For example, in the future, in the field of home security, the smart door sensor can be combined with a security system to grasp the dynamics of windows and doors through a mobile phone.

The Combination of IoT with Big Data. The combination of both will enhance the competitiveness of enterprises and government management services. Based on IoT big data technology can dig out the information and knowledge hidden in massive data to provide the basis for social and economic activities of mankind, so as to improve the operational efficiency in all fields and greatly increase the overall social and economic intensification ${ }^{[17][18]}$.

The Combination of IoT with Cloud Computing. Cloud Computing is the vanguard of turning to the IoT era in the Internet era. It takes the lead in completing the data processing technologies in the core technologies of IoT. At the same time, it can make statistics and analysis on big data to improve the utilization of resources in economic activities and reflect the consistency of consumption and demand ${ }^{[19]}$. In the future, more and more data will be collected and fed back to the Internet, and the IoT will be more widely used depending on cloud computing ${ }^{[20]}$.

IoT Will Become a Federal Environment. First, IoT equipment generates big data with unprecedented speed, size and heterogeneity, and have the ability to create smart products and services that benefit businesses, industries, and society. Second, the IoT ecosystem consisting of IoT equipment, cloud services, and data analytics equipment will be owned, managed and operated by independent vendors. Therefore, the Internet of Things will become a federal environment ${ }^{[21]}$.

Of course, with the development of the times, one day in the future, IOT equipment will communicate with each other without human's intervention and can make their own choices ${ }^{[22]}$.

\section{Development of pump equipment monitoring based on IoT}

With the rapid development of computer technology, graphical human-computer interface technology and network technology, the Internet of Things is slowly penetrating into all fields. In terms of technology and feasibility, it is entirely possible to utilize the Internet of Things for real-time monitoring of pump equipment, data processing, fault diagnosis, data sharing, product updates, etc. Figure. 2 shows the IoT-based pump equipment monitoring system structure. 


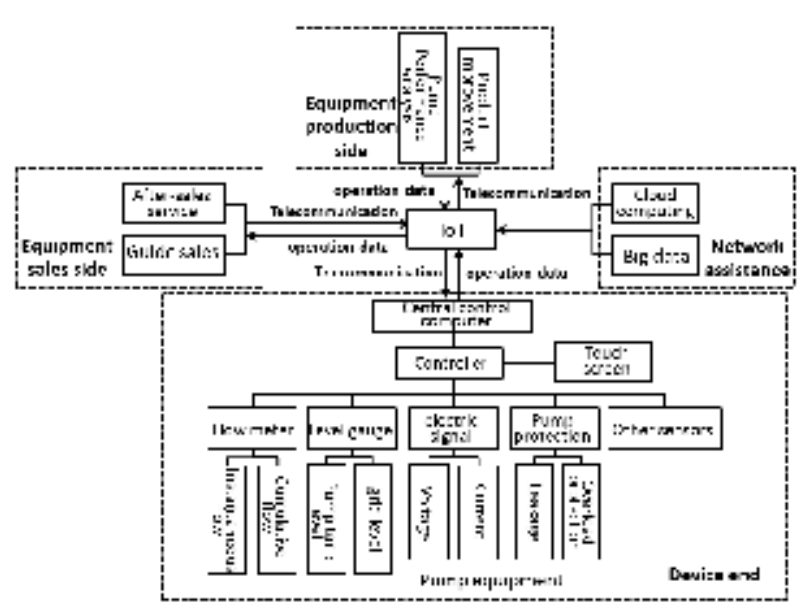

Figure. 2 the IoT-Based Pump Equipment Monitoring System Structure

It can be seen from the structure diagram that the three main parts (production end, sales end and application end) of the whole life cycle of the product are independent and integrated with each other. The data acquisition of the operation of the bottom pump equipment is realized by the sensor, central control computer monitors and diagnoses pump equipment, and data sharing is realized by network. Because network is a huge server, big data and cloud computing can make up for the shortcomings of low storage capacity, less data and slow processing speed of the local computer, thus strengthening the real time, sharing and data richness of the IoT monitoring. Sharing can send operating data of pump equipment back to the manufacturer in real time to guide the improvement of the product updates, and the sales end can also use the shared data to guide sales and after sales The perfect combination of pump equipment monitoring and IoT will enable lifecycle management of pump equipment and will be applied at all stages of lifecycle management.

Fault diagnosis technology for the pump equipment monitoring system will also be intelligent and real-time with the combination of IoT. Monitoring system and fault diagnosis technology are inseparable relationship. During pump equipment monitoring, the pump equipment may experience a variety of faults that have been found and are not found. In the IoT environment, the monitoring system sends the operating parameters and operating status of pumps to the fault management system in real time. Then, the fault management system can automatically call the stored fault modes to determine the type of fault, provide the measures and timely eliminate the fault. In the face of unselected faults, the fault management system exchanges information with other fault management systems through the network, self-learns and memorizes the fault, which will reduce the number of downtimes, increase production efficiency, and reduce the operator requirements. IoT makes pump monitoring field realize full intelligence in real sense.

\section{Conclusions}

With the rapid development of IoT technology, the world has entered an era of informationization. The extensive application of information technology has not only changed people's lifestyles, but also promoted the progress and development in all fields of society. IoT gradually applied to all areas of production and life. The integration of pump monitoring technology and IoT technology is an unprecedented opportunity for pump equipment monitoring, which will enable the intelligent development of pump equipment monitoring. Hope that in the future, the two can be more fully integrated, and gradually solve the current problems and deficiencies of IOT technology in the field of pump equipment monitoring, so that IoT better serve the field of pump equipment monitoring. 


\section{References}

[1] Dong-Fang Lv, Lei-Zhen Song. Application of Internet of Things in Drainage System of City Pump Statio[J]. Journal of Hunan Institute of Science and Technology (Natural Science Edition), 2013, 26(03): 49-52.

[2] Zhen-Yang Guan. Analysis on the Connection between Internet and Internet of Things and Its Development Trend[J]. Computer Knowledge and Technology: Academic Exchanges, 2016, 12(1X): 276-278.

[3] Ling Yin. Talking about the Connection and Development Status of Internet and Internet of Things [J / OL]. Electrical and Mechanical Engineering Technology, 2017, (S1): 189-191.

[4] Yi-Ke Feng. Development of Internet of Things Technology[J]. Journal of Information and Computer (Theory), 2016, (15): 166-167.

[5] Chen Zhang. The Development Process of Internet of Things Industry and Its Current Development Trend [J]. Mechatronics, 2011,17 (08): 4-9.

[6] OBDLink MX WiFi. http://www.obdlink.com/mxwf/. Retrieved Jun 2016

[7] VOLVO Autopilot http://www.volvocars.com/intl/About/Our-Innovation-Brands/IntelliSafe/IntelliSafe-Autopilot. Accessed June 2016

[8] Quirky EGG MINDER User Manual(http://cache.smarthome.com/manuals/89827-man.pdf or http://www.manualslib.commanual/683284/Quirky-Egg-Minder.html).Accessed May 2016

[9] Garmin Vivofit 2:https://buy.garmin.com/en-US/US/into-sports/health-fitness/previous_models_activity tracki ng/vivofit-2/prod504038.html. Retrieved Jun 2016

[10]Proteus Smart Pill. http://www.proteus.com/how-it-works/. Retrieved Jun 2016

[11]Jia Lu. PLC-based Emulsion Pump Intelligent Monitoring System [D]. Southwest Jiaotong University, 2009.

[12]Ning Huo. Development Trend of Pump Station Monitoring System and Its Structure [J]. Hydropower Automation and Dam Monitoring, 2004, (04): 81-85.

[13]Zhong-Yin Pan. Design and Verification of Turbine Pump Status Real-Time Monitoring System[D]. National University of Defense Technology, 2008.

[14]Zhen-Yu Wang. Design and Dynamic Optimization of Metering Pump Network Monitoring Platform[D]. Zhejiang University of Technology, 2014.

[15] Gartner Press Release, November 10, 2015. http://www.gartner.com/newsroom/id/3165317

[16] Georgakopoulos, D., \& Jayaraman, P. P. (2016). Internet of things: From internet scale sensing to smart services. Computing. Archives for Informatics and Numerical Computation, 98(10), 1041-1058.

[17] Hua-Wei Chen. Application of Integration of Internet of Things and Big Data[D]. Nanjing University of Posts and Telecommunications, 2015.

[18] Wen-Na He. Geographic Informationization Based on Internet of Things and Cloud Computing in Big Data Era [D] .Jilin University, 2013.

[19] Sheng-Ye Zhang. Research on IoT System of Spatial Health Monitoring Based on Cloud Computing [D] .Hangzhou: Zhejiang University, 2016. 
[20]Yang Xie. Modern Agricultural Internet of Things Monitoring System Based on Cloud Computing [D]. Southwest Jiaotong University, 2015.

[21]Zhen Hu. Problems and Development Strategies in the Application of Internet of Things Technology [J]. Private Higher Education Research, 2013, 03(09): 84-87.

[22] Ashton K (2009) That “Internet of things' thing”. In the real world, things matter more than ideas. Retrieved Jul 2015 